\title{
Efficacy of Switching from Infliximab to Subcutaneous Golimumab in Patients with Rheumatoid Arthritis to Control Disease Activity or Adverse Events
}

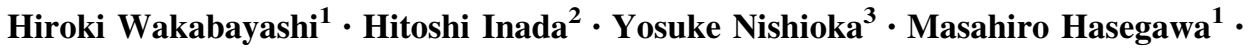 \\ Kusuki Nishioka $^{4} \cdot$ Akihiro Sudo $^{1}$
}

Published online: 26 December 2016

(C) The Author(s) 2016. This article is published with open access at Springerlink.com

\begin{abstract}
Background Some rheumatoid arthritis (RA) patients initially respond to treatment with infliximab (IFX), but subsequently their responsiveness decreases.

Objectives Our objective was to evaluate the efficacy and safety of switching from IFX to subcutaneous golimumab (GLM-SC) in RA patients.

Methods Thirty-three patients who had been treated for a mean 4.4 years with IFX (3-6 mg/kg/8 weeks) were switched to GLM-SC to control disease activity or adverse events. The patients with low disease activity (LDA) or remission were divided into two groups: the LDA group and the LDA every 8 weeks ( $q 8 \mathrm{w}$ ) group, which included patients with LDA or remission who switched to GLM therapy with $50 \mathrm{mg}$ at 4 - and 8 -week intervals, respectively. The moderate disease activity (MDA) group included patients with MDA who switched to GLM therapy with $50 \mathrm{mg}$ at 4-week intervals. Effects of the IFX to GLM-SC switch were evaluated at weeks 12, 24, and 52 after switching.
\end{abstract}

Results The mean disease activity score 28-ESR and -Creactive protein values in the LDA and LDAq8w groups

Hiroki Wakabayashi

whiroki@clin.medic.mie-u.ac.jp

1 Department of Orthopaedic Surgery, Mie University Graduate School of Medicine, 2-174 Edobashi, Tsu, Mie 514-8507, Japan

2 Department of Orthopaedic Surgery, Suzuka General Hospital, Suzuka, Mie, Japan

3 Clinical Research Institute for Rheumatic Disease, Shima, Mie, Japan

4 Institute of Medical Science, Tokyo Medical University, Tokyo, Japan were maintained from baseline throughout the 52-week treatment period. The mean disease activity score 28 values at 12, 24, and 52 weeks in the MDA group were improved significantly compared with baseline. Treatment discontinuations due to adverse events occurred in one patient in the MDA group, and no serious adverse events occurred during the observation period in the LDA group or the LDAq8w group. The GLM continuation rates at 52 weeks were $100 \%$ in the LDA and LDAq8w groups and $83.3 \%$ in the MDA group. Thus, GLM-SC treatment regimens were effective in controlling disease activity and improving the clinical response related to adverse events caused by IFX. Conclusion The clinical efficacy of GLM-SC was sustained or improved in patients who switched from IFX without serious safety concerns.

\section{Key Points}

Subcutaneous golimumab treatment regimens were effective in controlling disease activity and improving the clinical response related to adverse events caused by infliximab.

Administration of golimumab $50 \mathrm{mg}$ every 8 weeks may control disease activity if there is remission or low disease activity and a shorter disease duration.

\section{Introduction}

Biological therapies, especially tumor necrosis factor (TNF)- $\alpha$ inhibitors, have revolutionized the management of rheumatoid arthritis (RA). More than a decade has passed 
since the initial introduction of TNF inhibitors, which have greatly expanded treatment options for patients with RA who have not responded to other synthetic disease-modifying anti-rheumatic drugs [1].

Although the efficacy of this drug as a treatment for patients with active RA has been widely demonstrated $[2,3]$, some RA patients initially respond to treatment, but subsequently their responsiveness decreases [4]. One of the alleged reasons for this phenomenon is immunogenicity associated with the drug itself.

Infliximab (IFX) is a chimeric monoclonal antibody that specifically binds both soluble and membrane-bound TNF $\alpha$. It was the first anti-TNF $\alpha$ antibody that was clinically assessed for patients with RA. However, IFX can induce the formation of neutralizing antibodies [5], resulting in (secondary) loss of efficacy and the appearance of adverse effects such as infusion-related reactions $[6,7]$. In several recent studies, the retention rate of IFX was lower than of other TNF inhibitors. Thus, it is useful to switch to a less immunogenic biologic from IFX to control disease activity or adverse events.

Golimumab, a human anti-TNF monoclonal antibody, inhibits TNF bioactivity. In patients with RA who did not respond adequately to methotrexate (MTX) and/or antiTNF agents, subcutaneous golimumab (GLM-SC) plus MTX reduced RA signs/symptoms and was generally well tolerated [8-11]. GLM-SC is also less immunogenic than other TNF inhibitors [12, 13]. The purpose of this study was to evaluate the efficacy and safety of switching from IFX to GLM-SC in RA patients.

\section{Patients and Methods}

\subsection{Patients}

Data from patients with RA who were switched from IFX to GLM-SC therapy to control disease activity or because of the adverse events of IFX at Mie University and two other institutes were retrospectively analyzed. The Ethics Committee of Mie University approved the protocol for this study.

\subsection{Study Protocol}

The study was a simple observational study of patients after switching to GLM-SC to control disease activity or adverse events. Follow-up observation was monitored by symptoms, signs, and disease activity score (DAS) 28 at weeks $0,12,24$, and 52 .

\subsection{Golimumab Therapy}

In Japan, GLM-SC is required to be administered at 4-week intervals. In daily practice, however, the interval may be longer than 4 weeks, and no specific dosing interval has actually been established for GLM-SC. At our center, the decision on administration is made by the treating physician through discussion with each patient, considering the patient's general condition and convenience.

The patients with low disease activity (LDA) or remission were divided into two dose groups: [1] the LDA group, which included patients with LDA or remission who switched to GLM therapy with $50 \mathrm{mg}$ at 4-week intervals and [2] the LDA every 8 weeks ( $q 8 w$ ) group, which included patients with LDA or remission who switched to GLM therapy with $50 \mathrm{mg}$ at 8 -week intervals. The moderate disease activity (MDA) group included patients with MDA who switched to GLM therapy with $50 \mathrm{mg}$ at 4-week intervals.

\subsection{Clinical Assessment of Serum Markers}

The RA status was evaluated at 12, 24, and 52 weeks after the initiation of GLM treatment by the serum C-reactive protein (CRP) level and erythrocyte sedimentation rate (ESR). DAS 28-ESR and DAS28-CRP were used to evaluate RA disease activity compared with baseline; the DAS28 was calculated according to the standard formula $[14,15]$. The GLM continuation rates at 52 weeks were also examined. Data for patients who discontinued before week 52 were analyzed by the last observation carried forward method. For the safety evaluation, adverse events leading to discontinuation of treatment were assessed in each group.

\subsection{Statistical Analysis}

Differences between groups in terms of swollen and tender joint counts, patient global assessment, ESR, CRP, DAS28ESR, and DAS-CRP scores were assessed using the Wilcoxon signed rank test, Wilcoxon rank sum test, analysis of variance, Pearson's test, or the Tukey-Kramer honestly significant difference test. The last observation carried forward was applied when patients discontinued treatment or when data were unavailable. A $p$-value less than 0.05 was considered statistically significant.

\section{Results}

\subsection{Patients' Characteristics}

The subjects were 33 RA patients (26 were female; seven were male) who started receiving GLM treatment. Their baseline characteristics are summarized in Table 1. The mean age of the patients was 64.5 years, the mean disease duration was 11.8 years, and the mean duration of IFX treatment was 4.4 years. 
Table 1 Patients' characteristics

\begin{tabular}{|c|c|c|c|c|}
\hline & $\begin{array}{l}\text { LDA } \text { group }^{\mathrm{a}} \\
n=14\end{array}$ & 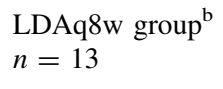 & $\begin{array}{l}\text { MDA group } \\
n=6\end{array}$ & $\begin{array}{l}p \text {-Value } \\
(\text { LDA vs. LDAq8w) }\end{array}$ \\
\hline Female, $[n(\%)]$ & $13(92.9)$ & $8(61.5)$ & $5(83.3)$ & 0.052 \\
\hline Age, years $(\mathrm{m} \pm \mathrm{SD})$ & $64.4 \pm 10.3$ & $63.2 \pm 12.4$ & $67.5 \pm 9.5$ & 0.784 \\
\hline RA disease duration, years $(\mathrm{m} \pm \mathrm{SD})$ & $16.0 \pm 10.7 * * * \#$ & $6.7 \pm 4.9$ & $13.3 \pm 11.9$ & $0.014 * * * \#$ \\
\hline Duration of IFX treatment, years (m \pm SD) & $5.4 \pm 2.4$ & $3.5 \pm 2.6$ & $4.0 \pm 3.1$ & 0.068 \\
\hline Steinbrocker sStage (I/II/III/IV), $n$ & $0 / 6 / 4 / 4$ & $0 / 7 / 6 / 0$ & $0 / 1 / 4 / 1$ & 0.163 \\
\hline Steinbrocker cClass $(1 / 2 / 3 / 4), n$ & $2 / 9 / 2 / 1$ & $0 / 11 / 2 / 0$ & $0 / 2 / 4 / 0^{*}$ & 0.962 \\
\hline Body weight, $\mathrm{kg}(\mathrm{m} \pm \mathrm{SD})$ & $51.4 \pm 6.6$ & $55.7 \pm 12.2$ & $54.1 \pm 11.2$ & 0.277 \\
\hline $\mathrm{BMI}(\mathrm{m} \pm \mathrm{SD})$ & $21.9 \pm 2.4$ & $22.5 \pm 3.1$ & $22.2 \pm 2.3$ & 0.603 \\
\hline $\mathrm{SJC}(\mathrm{m} \pm \mathrm{SD})$ & $0.4 \pm 0.7$ & $0.3 \pm 0.9$ & $2.5 \pm 3.6^{*}$ & 0.938 \\
\hline $\mathrm{TJC}(\mathrm{m} \pm \mathrm{SD})$ & $0.3 \pm 0.6$ & $0.3 \pm 0.6$ & $3.8 \pm 3.1^{*}$ & 0.967 \\
\hline $\mathrm{PGA}, \mathrm{mm}(\mathrm{m} \pm \mathrm{SD})$ & $20.9 \pm 22.0$ & $11.0 \pm 16.4$ & $36.8 \pm 25.3$ & 0.224 \\
\hline $\mathrm{ESR}, \mathrm{mm} / \mathrm{h}(\mathrm{m} \pm \mathrm{SD})$ & $14.0 \pm 9.3$ & $8.3 \pm 5.3$ & $30.5 \pm 21.9^{*}$ & 0.202 \\
\hline $\mathrm{CRP}, \mathrm{mg} / \mathrm{dLl}(\mathrm{m} \pm \mathrm{SD})$ & $0.13 \pm 0.21$ & $0.25 \pm 0.43$ & $0.96 \pm 1.20^{*}$ & 0.598 \\
\hline $\mathrm{RF}, \mathrm{IU} / \mathrm{mLl}(\mathrm{m} \pm \mathrm{SD})$ & $82.8 \pm 86.0$ & $146.2 \pm 162.4$ & $207.0 \pm 159.3$ & 0.236 \\
\hline DAS28-ESR $(m \pm S D)$ & $2.2 \pm 0.6$ & $1.7 \pm 0.8$ & $4.1 \pm 0.6^{*}$ & 0.064 \\
\hline DAS28-CRP $(m \pm S D)$ & $1.7 \pm 0.7$ & $1.6 \pm 0.5$ & $3.4 \pm 0.8^{*}$ & 0.819 \\
\hline Methotrexate use, $n(\%)$ & $14(100)$ & $13(100)$ & $5(83.3)$ & 0.999 \\
\hline Corticosteroid use, $n(\%)$ & $5(35.7)^{* * 8}$ & $12(92.3)$ & $6(100)$ & 0.004 \\
\hline
\end{tabular}

$B M I$ body mass index, $C R P$ C-reactive protein, $D A S 28$ disease activity score $28, E S R$ erythrocyte sedimentation rate, IFX infliximab, $L D A$ low disease activity, $m \pm S D$ mean \pm standard deviation, $M D A$ moderate disease activity, $P a G A$ patient's global assessment score, $q 8 \mathrm{w}$ every 8 weeks, $R A$ rheumatoid arthritis, $R F$ rheumatoid factor, The LDA group has a smaller number of patients with corticosteroid use than the LDAq8w group. The LDAq8w group has shorter RA disease duration than the LDA group. However, there are no significant differences in serum markers or disease activity between the LDA and LDAq8w groups at baseline

$T J C$, tender joint count, SJC, swollen joint count, TJC tender joint count,; PaGA, patient's global assessment score; ESR, erythrocyte sedimentation rate; CRP, C-reactive protein; RF, rheumatoid factor; DAS28, disease activity score $28 ; \mathrm{m} \pm \mathrm{SD}$, mean \pm standard deviation

$* p<0.05$ MDA group vs. LDA and LDAq8w; $* * \S p<0.05$ LDA group vs. LDAq8w group and MDA group; $* * * \# p<0.05$ LDA group vs. LDAq8w group

a The LDA group has a smaller number of patients with corticosteroid use than the LDAq8w group

b The LDAq8w group has a shorter RA disease duration than the LDA group. However, there are no significant differences in serum markers or disease activity between the LDA and LDAq8w groups at baseline

A total of 33 patients were analyzed during the survey period: 14 in the LDA group, 13 in the LDAq8w group, and 6 in the MDA group. One patient received GLM $50 \mathrm{mg} / 4$ weeks without MTX because of chronic kidney disease (nephritis). All the patients with LDA or remission (the LDA and the LDAq8w group) were switched to GLM therapy to control disease activity. In patients with MDA (the MDA group), three patients were switched to control disease activity and the adverse events of IFX. Adverse events with IFX were pyelonephritis in one patient and infusion reactions in two patients. The percentage of patients who used MTX concomitantly was $97.0 \%$ (32/33) overall, and 100, 100, and $83.3 \%$ in the LDA, LDAq8w, and MDA groups, respectively. The mean $( \pm$ standard deviation) dose of MTX in the LDA, LDAq8w, and MDA groups was $6.1( \pm 1.5) \mathrm{mg} /$ week, $5.8( \pm 1.5) \mathrm{mg} /$ week, and $6.8( \pm 1.1) \mathrm{mg} / \mathrm{week}$, respectively. The percentage of patients with concomitant corticosteroid use was $70.0 \%$
(23/33) of all patients. The percentage of patients who received corticosteroids in the LDA group $(35.7 \%)$ was significantly smaller than the percentage in the LDAq8w group $(92.3 \%)$ and the MDA group (100\%). The mean dose of corticosteroid in the LDA group as a whole was also significantly smaller compared with that in the LDAq8w group and the MDA group. However, the mean corticosteroid dose per patient who took a corticosteroid was not significantly different in the LDA $[3.5( \pm 1.4) \mathrm{mg} /$ day], LDAq8w $[4.5( \pm 1.5) \mathrm{mg} / \mathrm{day}]$, and MDA groups [5.8 $( \pm 2.0) \mathrm{mg} /$ day $]$.

The LDAq8w group had shorter RA disease duration than the LDA and MDA groups. There were no significant differences in serum markers or disease activity between the LDA and LDAq8w groups at baseline. The MDA groups were significantly worse in class, serum markers, and disease activity (except the patient's global assessment score) than the LDA and LDAq8w groups. 


\subsection{Effectiveness and Safety of Golimumab Therapy}

The mean DAS28-ESR and -CRP values in the LDA, LDAq8w, and MDA groups were maintained or improved from week 0 to week 52. Although the DAS28-ESR and CRP values showed no significant differences between the LDA and LDAq8w groups at baseline, DAS28-ESR at weeks 12,24 , and 52 was significantly lower in the LDAq8w group than in the LDA group. However, DAS28CRP was not significantly different between the two groups at each point throughout the 52-week treatment period (Table 2). In addition, the proportions of patients who achieved DAS28-ESR remission (defined as DAS28-ESR $<2.6)$ in the LDA group and the LDAq8w group went from $78.6 \%(11 / 14)$ and $76.9 \%(10 / 13)$ at week 0 to $100 \%(14 /$ 14 ) and $92.3 \%(12 / 13)$ at week 52 , respectively (Table 2 ). The proportion of patients who achieved DAS28-CRP remission $(<2.3)$ in the LDA group and the LDAq8w group over time went from $78.6 \%(11 / 14)$ and $92.3 \%(12 / 13)$ at week 0 to $100 \%(14 / 14)$ and $100 \%(13 / 13)$ at week 52, respectively (data not shown). Thus, GLM-SC treatment regimens were effective in maintaining and improving the clinical response achieved with LDA by IFX.

In the MDA group, the mean DAS28-ESR and -CRP values improved from baseline to 52 weeks. DAS28-ESR changes were significantly improved from baseline to week $12(p=0.025)$, week $24(p=0.011)$, and week 52 $(p=0.010)$, and DAS28-CRP changes were also significantly improved (week $52 ; p=0.030$ ). The proportions of patients who achieved remission and LDA (defined as DAS28-ESR <3.2) increased in a time-dependent manner. In three patients who switched to control disease activity, tow patients experienced remissions and one patient had MDA at week 52. There were no patients with flare to high disease activity.

The overall rate of treatment continuation was $100 \%$ at week 24 and $97.0 \%$ at week 52 . The rates of treatment continuation in the LDA, LDAq8w, and MDA groups were 100,100 , and $87.5 \%$, respectively, at week 52 . Reasons for discontinuation of GLM therapy included an adverse event in one patient after week 24 (Table 3).

\subsection{Adverse Events}

No patients in the LDA and LDAq8w groups withdrew from the study because of adverse events or lack of efficacy, while in the MDA group, one patient withdrew because of an adverse event (kidney cancer) after 24 weeks. No patients in the LDA and LDAq8w groups showed unexpected adverse events and discontinued GLM therapy by 52 weeks. However, in one patient, the MTX dose was decreased because of liver function test abnormalities and interstitial lung disease (Table 3). Infections

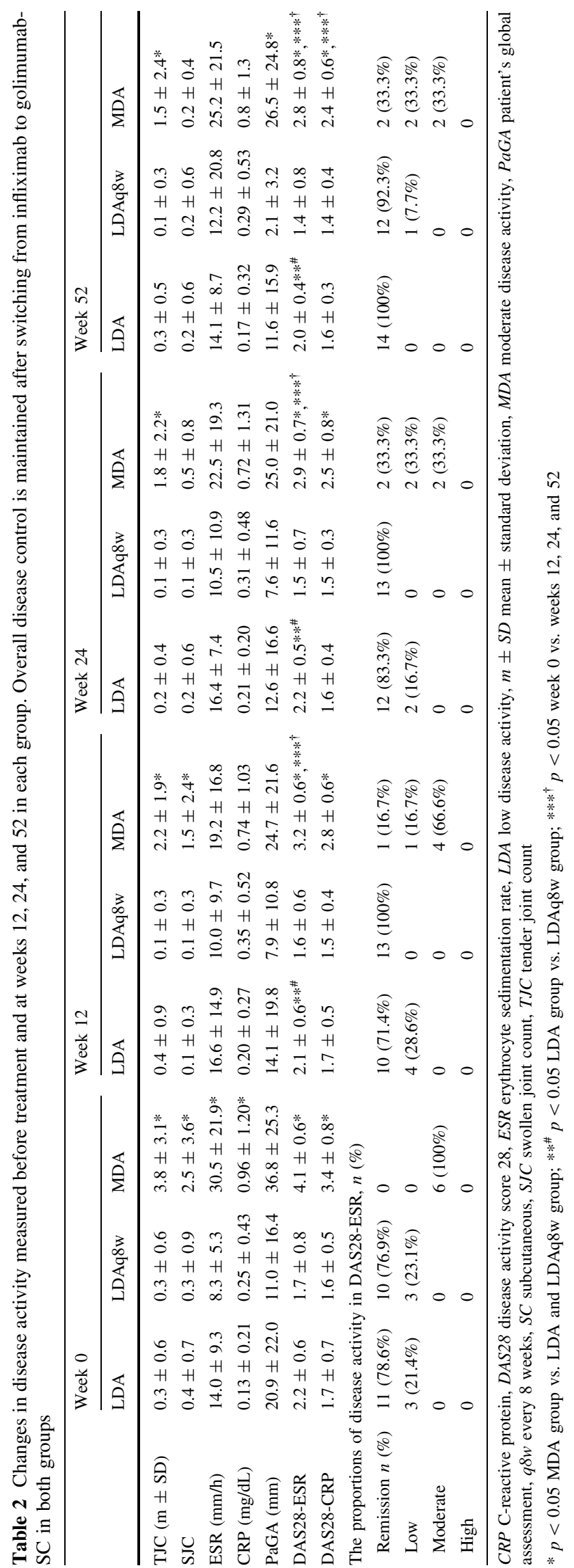


Table 3 Adverse events

\begin{tabular}{|c|c|c|c|}
\hline & $\begin{array}{l}\text { LDA group } \\
n=14\end{array}$ & $\begin{array}{l}\text { LDAq8w group } \\
n=13\end{array}$ & $\begin{array}{l}\text { MDA group } \\
n=6\end{array}$ \\
\hline Common cold & & 3 & 1 \\
\hline Upper respiratory tract infection & 1 & & \\
\hline Pyelonephritis & & & 1 \\
\hline Conjunctivitis & 1 & & \\
\hline Paronychia & & & 1 \\
\hline Vertigo & & 1 & \\
\hline Esophageal ulcer & & 1 & \\
\hline Eczema & & & 1 \\
\hline Kidney cancer & & & 1(discontinued GLM) ${ }^{\mathrm{a}}$ \\
\hline Interstitial lung disease & $1\left(\right.$ reduced MTX) ${ }^{\mathrm{b}}$ & & \\
\hline Liver function test abnormalities & $1\left(\right.$ reduced MTX) ${ }^{\mathrm{b}}$ & & \\
\hline Laboratory abnormality & 5 & 8 & 2 \\
\hline \multicolumn{4}{|c|}{$\begin{array}{l}G L M \text {, golimumab, } L D A \text { low disease activity, } M D A \text { moderate disease activity, } M T X \text { methotrexate, } q 8 w \\
\text { every } 8 \text { weeks, } S C \text { subcutaneous }\end{array}$} \\
\hline \multicolumn{4}{|c|}{$\begin{array}{l}\text { a The adverse event leading to discontinuation of GLM-SC is kidney cancer in one patient of the MDA } \\
\text { group }\end{array}$} \\
\hline
\end{tabular}

were the most commonly reported adverse events across all treatment groups, occurring in 14.3, 23.1, and $33.3 \%$ of patients in the LDA, LDAq8 week, and MDA groups, respectively (Table 3 ). Although no patient discontinued GLM because of infection, the infection rates were high. As a group with a high corticosteroid use, the rate of infectious disease was high. The infectious diseases may have been related to the use of corticosteroids.

\section{Discussion}

Biologic agents have enabled good therapeutic successes; however, the response to biologic therapy depends on treatment history and, especially, disease duration [16]. Some RA patients initially respond to treatment, but their responsiveness subsequently decreases [4]. This lack of clinical response in patients with antidrug antibodies (ADAbs) may be explained by an immune complex formation between TNF inhibitors and ADAbs suppressing the drug and restricting its therapeutic role. This may be related to an increase in the drug's clearance owing to the presence of immune complexes, which leads to lower serum drug concentrations $[6,17]$ or to direct neutralization of the biologic interfering with the fixation of the drug to TNF [18].

The reported rate of development of ADAbs to IFX in clinical studies ranges from 6 to $61 \%$ [13]. In a systematic review, $25.3 \%$ of patients using IFX developed ADAbs [12]. The presence of ADAbs toward IFX is generally associated with reduced serum IFX concentrations, with decreased clinical response to IFX and increased adverse events [19].

Among the newer anti-TNF agents, GLM is a human monoclonal anti-TNF agent administered subcutaneously every 4 weeks. Patients with active RA who previously received TNF inhibitor therapy and were treated with GLM and concomitant MTX in the GO-AFTER trial demonstrated clinically relevant improvement in disease activity and physical function after switching to GLM, regardless of which TNF inhibitor had been taken previously [11]. Of particular note, patients who switched from IFX appeared to show better subsequent responses to GLM. The MDA group had a significantly worse class than the LDA and LDAq8w groups and had longer RA disease duration than the LDAq8w group. Although only two of six MDA patients achieved remission, four patients achieved remission and LDA with switching to GLM. Two other patients were maintained with MDA, but they did not flare to high disease activity. The present study demonstrated clinical improvement of disease activity in the MDA group after switching to GLM and control of disease activity in the LDA group and the LDAq8w group.

In the previous intravenous GLM trial, therapy was administered at weeks 0 and 4 and q8w [20]. Clinical improvements were sustained through week 24 . However, the longer dosing interval in that trial yielded low systemic drug exposure in the later period of the 12-week dosing interval and did not result in a robust American College of Rheumatology 50 response at early time points [21]. Furthermore, antibodies to GLM were detected in a low percentage of patients (3\% at week 24) following repeated 
intravenous infusions of GLM q8w compared with every 12 weeks (5 and 7\% at weeks 24 and 48, respectively). Although an association between ADAb formation and lowered trough serum GLM levels has been reported in RA, no associations among ADAb formation and clinical response and adverse events were reported [9].

Administration of GLM $50 \mathrm{mg}$ q8w may control disease activity if there is remission or LDA and a shorter disease duration. Thus, these data evaluating two different intervals of administration of the same compound have demonstrated that response to GLM is maintained or improved, without an increase in toxicity/tolerability, following a switch from intravenous IFX to GLM-SC.

One limitation of the present study is the lack of radiographic data, which leaves the possibility that residual disease activity could induce structural damage, although we think that such effects, if they exist, are at best small. Second, we have no data on ADAbs. Third, the sample size was small. Finally, disease relapses after 12 months cannot be excluded.

\section{Conclusions}

The present results indicate that efficacy is adequately maintained in the majority of Japanese RA patients who switch from IFX q8w to GLM-SC q4-8w. Safety was also demonstrated to be consistent. These results provide supportive evidence for GLM-SC, as well as IFX, as useful options for treating RA.

Acknowledgements The authors thank Yukari Minami for the excellent assistance. This work was approved by the Institutional Review Board of Mie University.

\section{Compliance with Ethical Standards}

Funding No benefits or funds were received in support of the study.

Conflict of interest Hiroki Wakabayashi, Hitoshi Inada, Yosuke Nishioka, Masahiro Hasegawa, Kusuki Nishioka, and Akihiro Sudo declare that they have no conflicts of interest.

Open Access This article is distributed under the terms of the Creative Commons Attribution-NonCommercial 4.0 International License (http://creativecommons.org/licenses/by-nc/4.0/), which permits any noncommercial use, distribution, and reproduction in any medium, provided you give appropriate credit to the original author(s) and the source, provide a link to the Creative Commons license, and indicate if changes were made.

\section{References}

1. Hetland ML, Christensen IJ, Tarp U, et al. All Departments of Rheumatology in Denmark. Direct comparison of treatment responses, remission rates, and drug adherence in patients with rheumatoid arthritis treated with adalimumab, etanercept, or infliximab: results from eight years of surveillance of clinical practice in the nationwide Danish DANBIO registry. Arthritis Rheum. 2010;62(1):22-32.

2. Meroni PL, Valesini G. Tumour necrosis factor $\alpha$ antagonists in the treatment of rheumatoid arthritis: an immunological perspective. BioDrugs. 2014;28(Suppl 1):S5-13.

3. Richard-Miceli C, Dougados M. Tumour necrosis factor-alpha blockers in rheumatoid arthritis: review of the clinical experience. BioDrugs. 2001;15(4):251-9.

4. Weaver AL. The impact of new biologicals in the treatment of rheumatoid arthritis. Rheumatology (Oxford). 2004;43 Suppl. 3:iii17-23.

5. Wolbink GJ, Vis M, Lems W, et al. Development of antiinfliximab antibodies and relationship to clinical response in patients with rheumatoid arthritis. Arthritis Rheum. 2006;54(3):711-5.

6. van der Laken CJ, Voskuyl AE, Roos JC, et al. Imaging and serum analysis of immune complex formation of radiolabelled infliximab and anti-infliximab in responders and non-responders to therapy for rheumatoid arthritis. Ann Rheum Dis. 2007;66(2):253-6.

7. Bendtzen K, Geborek P, Svenson M, et al. Individualized monitoring of drug bioavailability and immunogenicity in rheumatoid arthritis patients treated with the tumour necrosis factor alpha inhibitor infliximab. Arthritis Rheum. 2006;54(12):3782-9.

8. Oldfield V, Plosker GL. Golimumab: in the treatment of rheumatoid arthritis, psoriatic arthritis, and ankylosing spondylitis. BioDrugs. 2009;23(2):125-35.

9. Kay J, Matteson EL, Dasgupta B, et al. Golimumab in patients with active rheumatoid arthritis despite treatment with methotrexate: a randomized, double-blind, placebo-controlled, dose-ranging study. Arthritis Rheum. 2008;58(4):964-75.

10. Keystone EC, Genovese MC, Klareskog L, et al. Golimumab in patients with active rheumatoid arthritis despite methotrexate therapy: 52-week results of the GO-FORWARD study. Ann Rheum Dis. 2010;69(6):1129-35.

11. Smolen JS, Kay J, Landewé RB, et al. Golimumab in patients with active rheumatoid arthritis who have previous experience with tumour necrosis factor inhibitors: results of a long-term extension of the randomised, double-blind, placebo-controlled GO-AFTER study through week 160. Ann Rheum Dis. 2012;71(10):1671-9.

12. Thomas SS, Borazan N, Barroso N, et al. Comparative immunogenicity of TNF inhibitors: impact on clinical efficacy and tolerability in the management of autoimmune diseases: a systematic review and meta-analysis. BioDrugs. 2015;29(4):241-58.

13. Vincent FB, Morand EF, Murphy K, et al. Antidrug antibodies $(\mathrm{ADAb})$ to tumour necrosis factor (TNF)-specific neutralising agents in chronic inflammatory diseases: a real issue, a clinical perspective. Ann Rheum Dis. 2013;72(2):165-78.

14. DAS-score. Available from: http://www.das-score.nl. Accessed 19 Dec 2016.

15. Wakabayashi H, Hasegawa M, Nishioka Y, et al. Which subgroup of rheumatoid arthritis patients benefits from switching to tocilizumab versus etanercept after previous infliximab failure?A retrospective study. Mod Rheumatol. 2012;22(1):116-21.

16. Smolen JS, Aletaha D. Rheumatoid arthritis therapy reappraisal: strategies, opportunities and challenges. Nat Rev Rheumatol. 2015;11(5):276-89.

17. Aarden L, Ruuls SR, Wolbink G. Immunogenicity of anti-tumor necrosis factor antibodies: toward improved methods of anti-antibody measurement. Curr Opin Immunol. 2008;20(4):431-5.

18. van Schouwenburg PA, van de Stadt LA, de Jong RN, et al. Adalimumab elicits a restricted anti-idiotypic antibody response in autoimmune patients resulting in functional neutralisation. Ann Rheum Dis. 2013;72(1):104-9. 
19. Pascual-Salcedo D, Plasencia C, Ramiro S, et al. Influence of immunogenicity on the efficacy of long-term treatment with infliximab in rheumatoid arthritis. Rheumatology (Oxford). 2011;50(8):1445-52.

20. Weinblatt ME, Bingham CO 3rd, Mendelsohn AM, et al. Intravenous golimumab is effective in patients with active rheumatoid arthritis despite methotrexate therapy with responses as early as week 2: results of the phase 3, randomised, multicentre, double- blind, placebo-controlled GO-FURTHER trial. Ann Rheum Dis. 2013;72(3):381-9.

21. Kremer J, Ritchlin C, Mendelsohn A, et al. Golimumab, a new human anti-tumor necrosis factor alpha antibody, administered intravenously in patients with active rheumatoid arthritis: fortyeight-week efficacy and safety results of a phase III randomized, double-blind, placebo-controlled study. Arthritis Rheum. 2010;62(4):917-28. 